\title{
Texture formation under rolling and primary recrystallization in $\mathrm{Fe}_{86} \mathrm{Ga}_{14}$ alloy
}

\author{
I. V. Gervasyeva, V.A. Milyutin ${ }^{\dagger}$ \\ †milutin@imp.uran.ru
}

M. N. Miheev Institute of Metal Physics, Ural Branch of RAS, 18 S. Kovalevskoy str., Yekaterinburg, 620137, Russia

$\mathrm{Fe}-\mathrm{Ga}$ alloys possess the increased magnetostriction in comparison with the traditional magnetostrictive materials Fe-Co and $\mathrm{Fe}-\mathrm{Al}$ and have a number of advantages over magnetostrictive materials based of rare earths elements. In literature, much attention is paid to composition $\mathrm{Fe}_{81} \mathrm{Ga}_{19}$, as this alloy possesses the greatest magnetostriction in one-phase area. Important condition for attainment of the best functional properties in this material is creation of certain crystallographic texture. However, the substantial difficulty at manufacture of samples and wares consists in a high brittleness of alloy at such content of gallium, for this reason the features of formation of crystallographic texture at deformation and recrystallization in this alloy are studied poorly. In this work, the attempt to study influence of various modes of rolling and annealing on structure and magnetic properties of Fe-Ga alloy with the smaller content of Ga is made. The structure and texture of primary recrystallization were studied by means of an electron back-scattering diffraction, besides field dependences of magnetization were measured. The amount of grains with the direction of easy magnetization in different samples was estimated by the value of magnetic induction at a defined value of magnetic field on a magnetization curve. The values of magnetostriction increased with increase of volume fraction of the components including the direction of easy magnetization of iron-gallium alloy. Absolute values of magnetostriction did not exceed $50 \mathrm{ppm}$ that is at the level of values for traditional materials, but lower than for $\mathrm{Fe}_{81} \mathrm{Ga}_{19}$. Plastic deformation by hot and, especially cold rolling causes great difficulties in connection with a high brittleness of alloy even with the lower value of gallium concentration. Therefore, it is necessary to find new methods of a plastic deformation of alloy or to add the chemical elements improving plastic properties of material.

Keywords: Fe-Ga alloys, rolling, recrystallization, crystallographic texture, magnetostriction.

УДК: 669-1

\section{Образование текстуры при прокатке и первичной рекристаллизации в сплаве $\mathrm{Fe}_{86} \mathrm{Ga}_{14}$}

\author{
И. В. Гервасьева, В. А. Милютин \\ Институт физики металлов имени М.Н. Михеева УрО РАН, ул. С. Ковалевской 18, Екатеринбург, 620137, Россия
}

Сплавы Fe-Ga обладают повышенной магнитострикцией по сравнению с традиционными магнитострикционными материалами $\mathrm{Fe}-\mathrm{Co}$ и $\mathrm{Fe}-\mathrm{Al}$ и имеют ряд преимуществ перед магнитострикционными материалами на основе редкоземельных элементов. В литературе большое внимание уделяется составу $\mathrm{Fe}_{81} \mathrm{Ga}_{19}$, поскольку при таком соотношении сплав обладает наибольшей магнитострикцией в однофазной области. Важным условием для достижения наилучших функциональных свойств в этом материале является создание определенной кристаллографической текстуры. Однако существенная проблема при изготовлении образцов и изделий состоит в высокой хрупкости сплава при таком содержании галлия, по этой причине особенности формирования кристаллографической текстуры при деформации и рекристаллизации в нём изучены слабо. В настоящей работе сделана попытка изучить влияние различных режимов прокатки и отжига на структуру и магнитные свойства Fe-Ga сплава с меньшим содержанием $\mathrm{Ga}$. Структура и текстура первичной рекристаллизации изучалась с помощью метода обратного электронного рассеяния, кроме этого строились полевые зависимости намагниченности. Доля зерен с направлением легкого намагничивания в разных образцах оценивалась по величине индукции при определенном значении магнитного поля на кривой намагничивания. Величина значений магнитострикции повышалась с возрастанием объемной доли компонент, включающих направление легкого намагничивания железо-галлиевого сплава. Абсолютные значения магнитострикции не превышали 50 ppm, что находится на уровне величин для традиционных материалов и существенно ниже значений магнитострикции в $\mathrm{Fe}_{81} \mathrm{Ga}_{19}$. Деформационная обработка с помощью и горячей и, особенно холодной прокатки вызывает большие трудности в связи с высокой хрупкостью сплава даже с пониженным значением концентрации галлия. Поэтому в дальнейшем нужно изыскивать новые методы пластической деформации сплава или добавлять химические элементы, улучшающие пластические свойства материала.

Ключевые слова: сплавы Fe-Ga, прокатка, рекристаллизация, кристаллографическая текстура, магнитострикция. 


\section{1. Введение}

В работе [1] было впервые показано, что при добавлении $\mathrm{Ga}$ в Fe происходит значительное увеличение магнитострикции. По сравнению с традиционными магнитострикционными материалами, такими как $\mathrm{Fe}-\mathrm{Co}$ и $\mathrm{Fe}-\mathrm{Al}$, величина магнитострикции $\mathrm{Fe}-\mathrm{Ga}$ в несколько раз выше, в монокристалле она может достигать $400 \times 10^{-6}$ [2]. По сравнению с магнетиками на основе редкоземельных элементов, которые обладают огромной магнитострикцией, данный сплав имеет ряд преимуществ, а именно низкие поля насыщения (около 200 Э в $\mathrm{Fe}-\mathrm{Ga}$ по сравнению с 1000 Э в Tb-Dy-Fe) [3], низкая стоимость, слабая зависимость магнитострикции от температуры (около $0.5 \mathrm{ppm} \mathrm{K}^{-1}$ ) [4] и более высокие механические свойства [5]. Сплавы $\mathrm{Fe}-\mathrm{Ga}$ имеют высокую точку Кюри $\left(650^{\circ} \mathrm{C}\right.$ [6]) и хорошую коррозионную стойкость [7], а также обладают хорошими демпфирующими свойствами благодаря магнитомеханической природе затухания внутреннего трения $[8,9]$. Примерно до 21 ат.\% Ga при закалке сплавы сохраняют решетку $\alpha$-Fe, кроме того, большую роль играет скорость охлаждения $[10,11]:$ при большей концентрации или медленном охлаждении появляются упорядоченные фазы. Авторы работ [12-15] показали, что при закалке с высоких температур в образцах Fe-Ga величина магнитострикции выше, чем в медленно охлажденных. В работе [3] показано монотонное повышение значений магнитострикции в направлении $<100>$ до $420 \times 10^{-6}$ при увеличении концентрации галлия в монокристалле до 21.4 ат.\%. Известно также, что магнитострикция существенным образом зависит от кристаллографической текстуры в материале. Показано, что в сплаве $\mathrm{Fe}_{81} \mathrm{Ga}_{19}$ величина магнитострикции может отличаться в несколько раз в зависимости от того, какое кристаллографическое направление преобладает [3]. В литературе наибольшее внимание уделяется составу $\mathrm{Fe}_{81} \mathrm{Ga}_{19}$, поскольку этот сплав обладает наибольшей магнитострикцией в однофазной области. Однако большая проблема при изготовлении образцов состоит в высокой хрупкости сплава $[16,17]$. В настоящей работе поставлена задача изучить особенности формирования кристаллографической текстуры и её влияние на магнитострикцию в сплаве с меньшим содержанием галлия. Определяющую роль в формировании кристаллографической текстуры играет способ обработки материала. В работе для варьирования конечной текстуры первичной рекристаллизации использовались разные режимы горячей и холодной прокатки и последующего отжига образцов из сплава $\mathrm{Fe}_{86} \mathrm{Ga}_{14}$.

\section{2. Эксперимент}

Сплав $\mathrm{Fe}-14$ ат.\% Ga был выплавлен методом электродугового переплава. В исходной структуре не выявлено какой-либо преимущественной ориентировки, средний линейный размер зерна составлял более 500 мкм. Слиток длиной 60 мм разрезался поперек длинной оси на 4 равные части. Короткий слиток ковали при $1100^{\circ} \mathrm{C}$ перпендикулярно оси слитка до 7-8 мм, затем подвергали горячей прокатке до 2.3 мм. Далее полосы прокатывались в холодную на лабораторном прокатном стане «Кварто-150» с диаметром рабочих валков 55 мм с разной степенью деформации. Все имеющиеся образцы подвергались либо однократной холодной прокатке, либо двукратной. Однократная холодная прокатка проводилась с 2.3 мм до толщин $0.53,0.7,0.9$ мм, степени деформации 77,70 и $60 \%$ соответственно. Двукратная холодная прокатка осуществлялась путём деформации $60 \%$, отжига и последующей деформации на 50 или $67 \%$ (до толщин 0.46 или 0.3 соответственно). В некоторых случаях направление второй холодной прокатки было перпендикулярно направлению горячей и первой холодной прокатки. Степень деформации измерялась по относительному изменению толщины листа. Окончательный рекристаллизационный отжиг проводился в вакууме при температуре 800,900 или $1000^{\circ} \mathrm{C}$ в течение 15 или 30 минут. Целью реализации разных способов прокатки и отжига было исследование их влияния на тип текстуры рекристаллизации, а в дальнейшем - на значения магнитострикции. Структура и текстура всех рекристаллизованных образцов определялась методом дифракции обратно рассеянных электронов (EBSD) на сканирующем электронном микроскопе FEI “Quanta 200” с EBSD приставкой фирмы EDAX. C помощью сопутствующего программного обеспечения для каждой съемки строились ориентационные карты для выбранного участка образца, картины качества расшифровки картин Кикучи (“Image Quality”), полные полюсные фигуры, функция распределения ориентаций (ФРО), рассчитывались объемные доли отдельных ориентировок с отклонением $\pm 10^{\circ}$. В рекристаллизованных образцах определялась магнитострикция и строились полевые зависимости намагниченности. Для этого был использован вибромагнитометр Lakeshore 7407 VSM и стандартный метод определения магнитострикции с помощью тензодатчиков, длина тензодатчиков составляла 5 мм.

\section{3. Результаты и обсуждение}

Режимы прокатки, температура и длительность отжигов образцов, обработанных по 7 вариантам, представлены в Табл. 1. В этой же таблице приведены сечения ФРО при $\varphi 2=45^{\circ}$, максимальное значение ФРО, объемная доля компонент, содержащих направление легкого намагничивания, полученные из данных EBSD для рекристаллизованных образцов.

Из всех исследованных образцов для дальнейшего изучения были выбраны три (№№ 1, 3 и 6), с наиболее высокой объемной долей на их поверхности зерен с кристаллографическими плоскостями $\{100\}$ и $\{110\}$, содержащими направление легкого намагничивания $<100>$. При расчете учитывались плоскости с отклонением от поверхности до $\pm 10^{\circ}$. На Рис. $\left.1 \mathrm{a}-1 \mathrm{c}\right)$ приведены полные ориентационные карты для этих образцов.

В образце №6, было обнаружено крупное зерно с ориентировкой, близкой к плоскости $\{110\}$. Направление $<100>$ в этом зерне близко к направлению прокатки (см. расположение значка кристаллической решетки на Рис. 1с). Однако, как показало исследование точной разориентации плоскости $\{110\}$ с плоскостью прокатки 
Табл. 1. Режимы холодной прокатки (ХП) и отжига образцов Fe-14 ат. \% Ga, предварительно подвергнутых горячей прокатке (ГП), их структура и текстура (ФРО), полученные методом EBSD.

Table 1. Modes of cold rolling (CR) and annealing of pre-hot rolled (GP) Fe-14 at. \% Ga samples, their structure and texture (ODF) from the EBSD measurements.

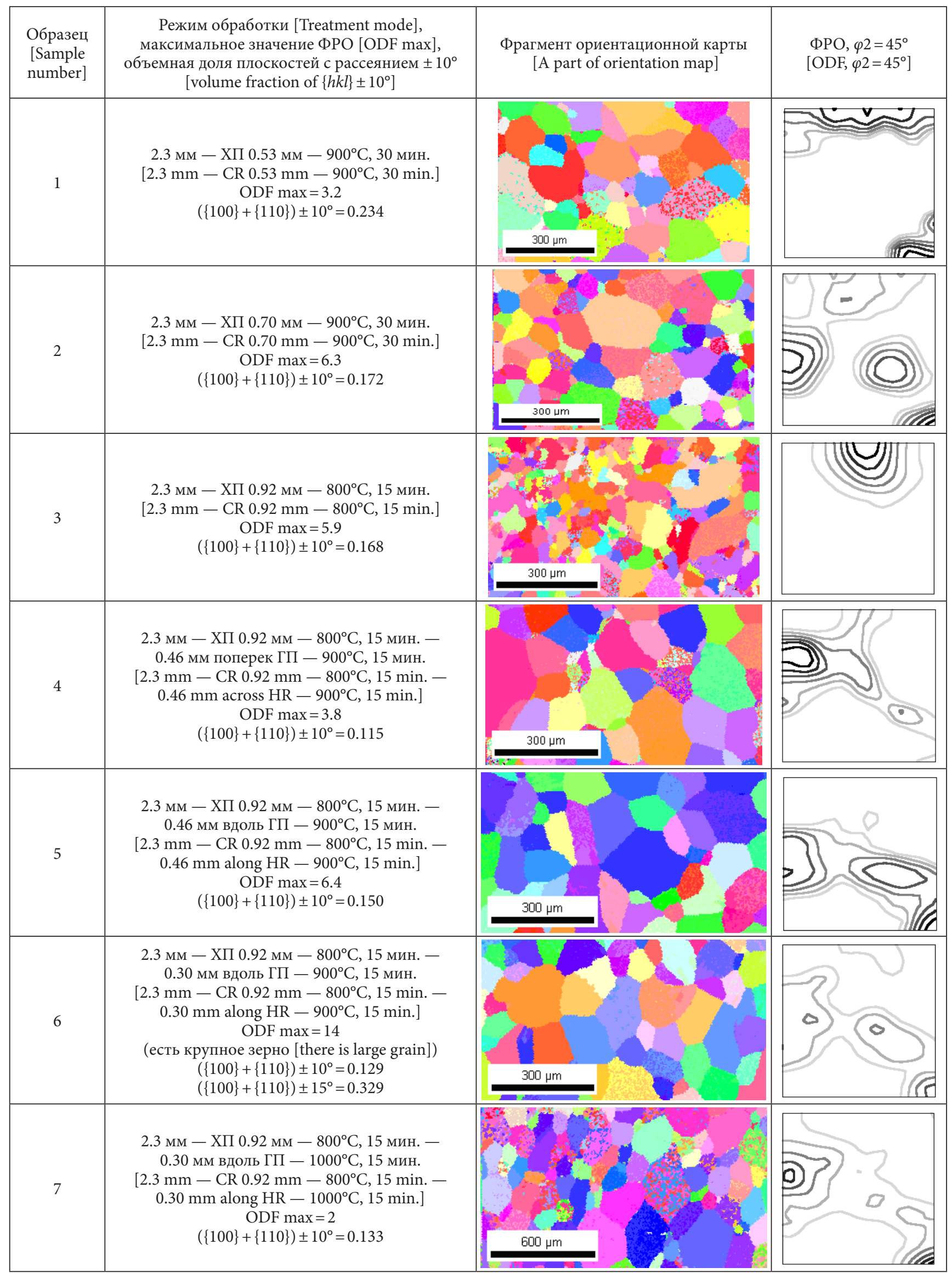




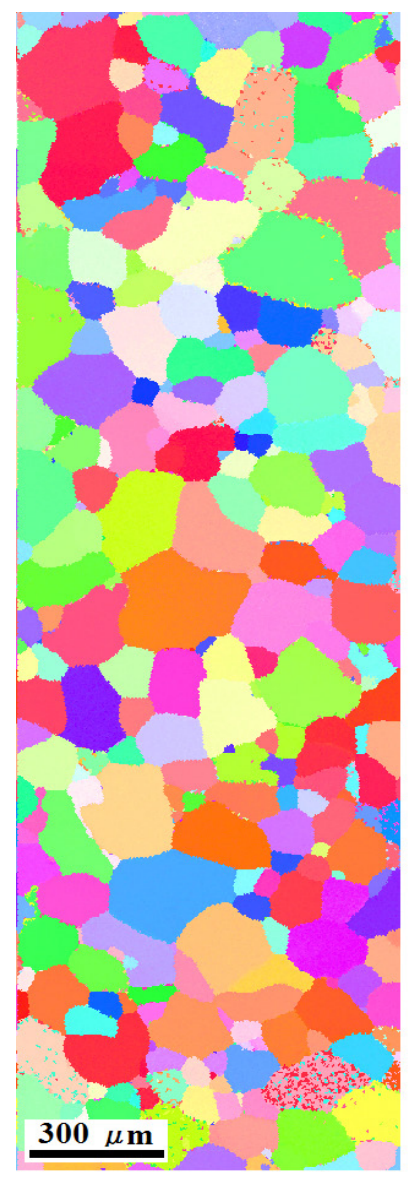

a

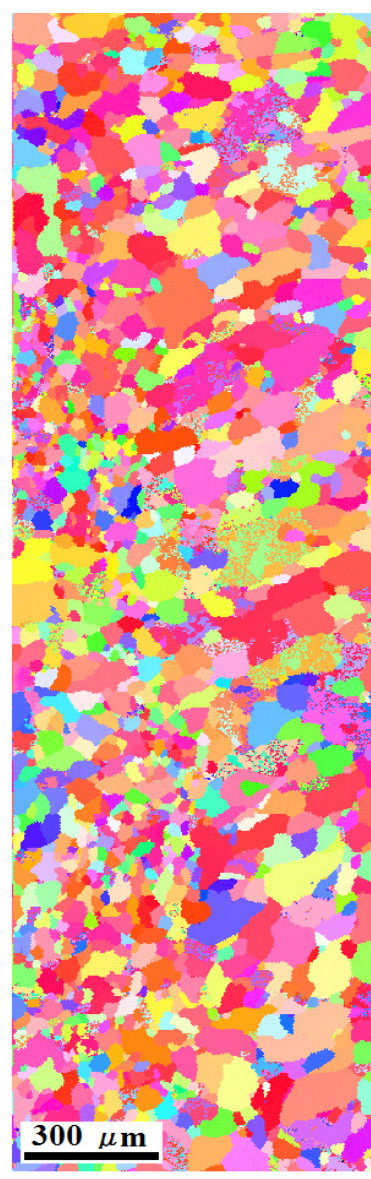

$\mathrm{b}$

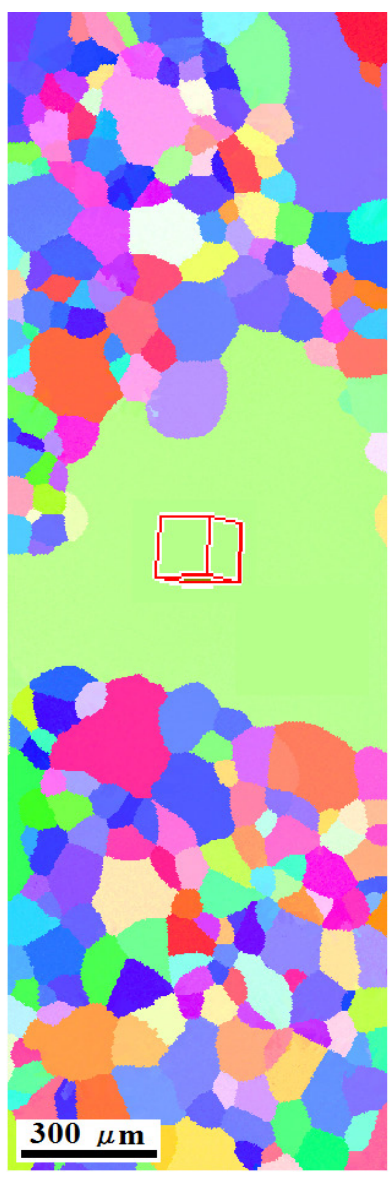

c
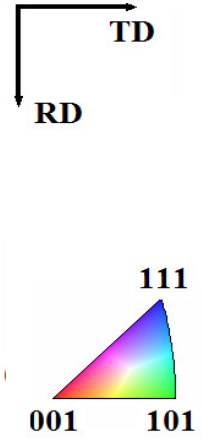

d

Рис. 1. Ориентационные карты EBSD с образцов № 1 (a), 3 (b) и 6 (c), полученных разными способами деформации и отжига; изображение ориентировок в плоскости образца на стереографическом треугольнике (d). Направление исходной прокатки вертикально.

Fig. 1. Orientation EBSD maps of the samples No 1 (a), 3 (b) и 6 (c) after different modes of deformation and annealing and orientation image on the sample plane, shown by stereographic triangle (d). A direction of initial rolling is vertical.

в программе EBSD, эта разориентировка составляет $15^{\circ}$. В Табл. 2 приведены значения объемных долей кристаллографических плоскостей разного типа в отожженных образцах. Для образца № 6 приведены значения объемной доли с разным рассеянием. Наибольшее содержание кристаллографических компонент $\{100\}$ и $\{110\}$ наблюдается в образце, подвергнутом однократной холодной прокатке на $77 \%$ и отжигу 30 минут при $900^{\circ} \mathrm{C}$. Таких значений температуры и времени выдержки достаточно, чтобы сформировалась равномерная структура рекристаллизации в отличие от образца № 3 .

Значение индукции насыщения составило в среднем 1.7 T, что примерно соответствует данным, имеющимся в литературе для сплавов $\mathrm{Fe}-\mathrm{Ga}$ соответствующего состава [1]. В Табл. 3 приведены значения магнитной индукции в поле до насыщения 100 Ое или 7960 A/m $\left(\mathrm{B}_{7960}\right)$, полученные из построенных петель гистерезиса при приложении магнитного поля вдоль направления прокатки, а также значения магнитострикции.

Величина индукции в фиксированном магнитном поле до насыщения косвенно показывает количество элементов структуры с направлениями, близкими к направлению легкого намагничивания. Направление легкого намагничивания <001> содержится в кристаллографи- ческих плоскостях $\{100\}$ и $\{110\}$. Как видно из Табл. 2 и 3 , изменение магнитной индукции в поле до насыщения следует за изменением объемной фракции этих плоскостей с учетом большого зерна в образце № 6. При измерении магнитострикции тензодатчик, вероятно, не попал на крупное зерно, и значение магнитострикции соответствует объемной доле мелких зерен с соответствующим рассеянием, которая в этом образце является наименьшей. Необходимо отметить, что разница в значениях магнитострикции является не многим больше ошибки измерения. Абсолютные значения магнитострикции также невелики, что объясняется в первую очередь низкой концентрацией галлия в исследованном сплаве, однако эти значения находятся на уровне значений магнитострикции в традиционных материалах.

\section{4. Заключение}

Величина значений магнитострикции в прокатанном и отожженном сплаве $\mathrm{Fe}_{86} \mathrm{Ga}_{14}$ повышается с возрастанием объемной доли компонент, включающих направление легкого намагничивания сплава. Наиболее благоприятная с точки зрения магнитострикции текстура сформировалась при однократной холодной прокатке 
Табл. 2. Значения объемной доли кристаллографических плоскостей $\{h k l\}$ в рекристаллизованных образцах. Table 2. The volume fraction of crystallographic planes in recrystallized samples.

\begin{tabular}{|c|c|c|c|c|c|c|}
\hline \multirow{2}{*}{$\begin{array}{c}\text { Образец } \\
\text { [Sample number] }\end{array}$} & \multicolumn{6}{|c|}{$\begin{array}{l}\text { Тип кристаллографической плоскости, рассеяние от плоскости образца до } \pm 10^{\circ} \\
\left.\text { [The type of crystallographic plane, spread about the sample plane } \pm 10^{\circ}\right]\end{array}$} \\
\hline & $\{100\}$ & $\{110\}$ & $\{100\}+\{110\}$ & $\{111\}$ & $\{112\}$ & $\{113\}$ \\
\hline 1 & 0.12 & 0.12 & 0.24 & 0.02 & 0.10 & 0.11 \\
\hline 3 & 0.13 & 0.04 & 0.17 & 0.02 & 0.07 & 0.20 \\
\hline 6 & 0.04 & $\begin{array}{c}0.09 \\
0.33\left( \pm 15^{\circ}\right)\end{array}$ & $\begin{array}{c}0.13 \\
0.37\left( \pm 15^{\circ}\right)\end{array}$ & 0.11 & 0.14 & 0.08 \\
\hline
\end{tabular}

Табл. 3. Магнитные свойства исследованных образцов.

Table 3. Magnetic properties of the investigated samples.

\begin{tabular}{|c|c|c|}
\hline $\begin{array}{c}\text { Образец } \\
\text { [Sample number] }\end{array}$ & $\begin{array}{c}\text { Магнитная индукция в поле } 100 \text { Э }(7960 \mathrm{~A} / \mathrm{m}), \mathrm{B}_{7960}, \text { Тл } \\
\left.\text { [Magnetic induction in the field of } 100 \mathrm{Oe}(7960 \mathrm{~A} / \mathrm{m}), \mathrm{B}_{7960}, \mathrm{~T}\right]\end{array}$ & $\begin{array}{c}\text { Магнитострикция, } \lambda, \text { ррт } \\
\text { [Magnetostriction, } \lambda, \text { ppm] }\end{array}$ \\
\hline 1 & 0.17 & 47 \\
\hline 3 & 0.08 & 41 \\
\hline 6 & 0.27 & 36 \\
\hline
\end{tabular}

на $77 \%$ и отжиге при $900^{\circ} \mathrm{C}$. Механическая обработка с помощью и горячей и, особенно холодной прокатки вызывает большие трудности в связи с высокой хрупкостью сплава даже с пониженным значением концентрации галлия. Поэтому в дальнейшем нужно изыскивать новые методы пластической деформации сплава или добавлять химические элементы, улучшающие пластические свойства материала, а также искать способы создания текстуры близкой к кубической. Значительный вклад может внести, например, проведение отжига в сильном магнитном поле. Как показано, например, в $[18,19]$ такая обработка способствует формированию текстурных компонент с направлением легкого намагничивания в магнитомягких материалах.

Благодарность/Acknowledgements. Работа выполнена в рамках государственного задания ФАНО России "Магнит» № АААА-А18-118020290129-5 при частичной поддержке РФФИ (проект №18-03-00623)./The research was carried out within the state assignment of FASO of Russia (theme "Magnet" No. AAAA-A18-118020290129-5), supported in part by RFBR (project No. 18-03-00623).

\section{Литература/References}

1. A.E. Clark, J. B. Restorff, M. Wun-Fogle, T. A. Lograsso, D. L Schlagel. IEEE Transactions on Magnetics. 36, 3238 (2000). DOI: 10.1109/20.908752

2. Q. Xing, Y. Du, R.J. McQueeney, T.A. Lograsso. Acta Materialia. 56, $4536 \quad$ (2008). DOI: 10.1016/j.actamat.2008.05.011

3. S. Na, A.B. Flatau. Scripta Materialia. 66, 307 (2012). DOI: 10.1016/j.scriptamat.2011.11.020

4. R.A. Kellogg, A.B. Flatau, A.E. Clark, M. WunFogle, T.A. Lograsso, J. Appl. Phys. 91, 7821 (2002). DOI: $10.1063 / 1.1452216$

5. J. Atulasimha, A. B. Flatau. Smart Mater. Struct. 20, 043001 (2011). DOI: 10.1088/0964-1726/20/4/043001

6. R.A. Kellogg. Development and modeling of iron- gallium alloys. PhD Thesis Engineering Mechanics, Iowa State University, Ames, Iowa (2003).

7. T. V. Jayaraman, N. Srisukhumbowornchai, S. Guruswamy, M.L. Free. Corros. Sci. 49, 4015 (2007). DOI: $10.1016 /$ j.corsci.2007.05.010

8. I.S. Golovin, A. Rivière. Intermetallics. 19, 453 (2011). DOI: $10.1016 /$ j.intermet.2010.10.017

9. I.S. Golovin, J. Cifre. Journal of Alloys and Compounds. 584, 322 (2014). DOI: 10.1016/j.jallcom.2013.09.077

10. A.E. Clark, M. Wun-Fogle, J.B. Restorff, T. A. Lograsso, J.R. Gullen. IEEE Transaction on magnetics. 37, 2678 (2001). DOI: 10.1109/20.951272

11. A.E. Clark, K. B. Hathaway, M. Wun-Fogle, J.B. Restorff, T. A. Lograsso, M. Keppens, G. Petculescu, R. A. Taylor. J. Appl. Phys. 93, 8621 (2003). DOI: 10.1063/1.1540062

12. Q. Xing, T. A. Lograsso. Scripta Materialia. 65, 359 (2011). DOI: $10.1016 /$ j.scriptamat.2011.05.010

13. J. Zang, T. Ma, M. Yan. Physica B. 405, 3129 (2010). DOI: $10.1016 /$ j.physb.2010.04.027

14. C. Mudivarthi, M. Laver, J. Cullen, A. B. Flatau, V. Wuttig, Journal of Applied Physics. 107, 09A957 (2010). DOI: $10.1063 / 1.3359814$

15. M. V. Petrik, O.I. Gorbatov, Yu.N. Gornostyrev, JETP Letters. 98, 809 (2014). (in Russian) [М.В. Петрик, О.И. Горбатов, Ю.Н. Горностырев. Письма в ЖЭТФ. 98(12), 912 (2013).]

16. A. Sun, J. Liu, C. Jiang, J. Mater. Sci. 49, 4565 (2014). DOI: $10.1007 / \mathrm{s} 10853-014-8156-9$

17. J. Li, X. Gao, J. Zhu, J. Li, M. Zhang. Journal of Alloys and Compounds. 484, 203 (2009). DOI: $10.1016 /$ j.jallcom.2009.03.008

18. V.A. Milyutin, I. V. Gervasyeva. Letters on Materials. 8(1), 59 (2018). (in Russian) [В.А. Милютин, И. В. Гервасьева. Письма о материалах. 8(1), 59 (2018).] DOI: 10.22226/2410-3535-2018-1-59-65

19. M.Z. Salih, M. Uhlarz, F. Pyczak, H.-G. Brokmeier, B. Weidenfeller, N. Al-hamdany, W. M. Gan, Z. Y. Zhong, N. Schell. Journal of Magnetism and Magnetic Materials. 381, 350 (2015). DOI: 10.1016/j.jmmm.2015.01.004 